Information about the course of COVID-19 in patients with certain rheumatic diseases is still lacking

Objectives: To examine clinical course of COVID-19 in patients with Sjogren's syndrome treated with anti-CD20 monoclonal antibody (rituximab).

Methods: Single center observational study. Diagnosis of SjS was based on ECR/EULAR 2016 criteria. COVID-19 diagnosis was based on positive PCR test even without clinical symptoms and/or typical clinical features (CT signs, fever and anosmia). Rituximab was administrated in two $1000 \mathrm{mg}$ infusions 14 days apart for the $1^{\text {st }}$ course, then $500 \mathrm{mg}$ every 6 months.

Results: 19 patients were included, 18 women and 1 man. Median age was 55 years (29-70 years), and median rituximab treatment duration was 24 months (1-48 months). Five patients had concomitant RA (2 patients), SLAE (1 pt), Systemic sclerosis (2 patients). Patients with RA took baricitinib and methotrexate as well. 3 patients had MALT-lymphoma anamnesis $(24,38$ and 24 months before the diagnosis of COVID-19). Only 3 patients had chronic ischemic heart disease and/or arterial hypertension. 12 patients were PCR positive, 6 negative and in 1 the test was not done. 11 patients had full and 4 partial B-cell depletion in peripheral blood. Five patients had $<20 \%$ lung involvement on CT, 2 patients $-20-40 \%$ and 4 patients $-40-60 \%$. Three patients with $40-60 \%$ lung involvement required hospitalization due to marked shortness of breath and long febrile period, 2 of them received anti-IL6 treatment and neither of them required mechanical lung ventilation (either non-invasive or invasive). Seventeen patients were treated at home and recovered in 10-24 days. Anti-SARS-CoV-2 IgG were measured in 9 patients, $6(66.7 \%)$ of them were positive.

Conclusion: It seems that neither SjS itself nor anti-CD20 therapy predisposes patients to severe course of COVID-19. Presumably risk factors such as age, diabetes or anamnesis of cardiovascular diseases have far more significant impact on COVID-19 severity. Data hints that anti-CD20 therapy might negatively affect the formation of specific anti-SARS-CoV-2 humoral immunity, but further investigation is required to determine that with any degree of certainty.

Disclosure of Interests: None declared

DOI: 10.1136/annrheumdis-2021-eular.2933

\section{POS1227 IMPACT OF A PRE-EXISTING INTERSTITIAL LUNG DISEASE ON SEVERITY OF COVID-19 IN PATIENTS WITH RHEUMATIC AND MUSCULOSKELETAL DISEASES}

P. A. Juge ${ }^{1}$, E. Hachulla ${ }^{2}$, C. Richez ${ }^{3}$, E. Drumez ${ }^{4}$, A. Duhamel ${ }^{4}$, R. Borie ${ }^{5}$, P. Dieudé ${ }^{1} .{ }^{1}$ Bichat-Claude Bernard Hospital, Rheumatology, Paris, France; ${ }^{2}$ Chu De Lille, Internal Medecine, Lille, France; ${ }^{3}$ Hospital Center University De Bordeaux, Rheumatology, Bordeaux, France; ${ }^{4}$ Chu De Lille, Biostatistic, Lille, France; ${ }^{5}$ Bichat-Claude Bernard Hospital, Pulmonology, Paris, France

Background: COVID19 due to SARS-CoV-2 infection implies an important anti-viral immune response leading to a major inflammatory syndrome with increased pro-inflammatory cytokine levels (i.e. the cytokine storm paradigm). The impact of a preexisting interstitial lung disease (ILD) on the morbi-mortality of COVID-19 is unclear. An increased mortality rate has been identified in studies that included a limited number of patients with ILD from various etiologies. To date, no studies have investigated the severity of COVID-19 in patients with preexisting ILD in a large population of rheumatic and musculoskeletal diseases (RMD-ILD). Since March 18th, 2020, the French RMD COVID19 dataset (NCT04353609) includes patients with an RMD and SARS-Cov-2 infection.

Objectives: To assess the impact of a preexisting ILD on COVID-19 morbi-mortality within the French RMD COVID-19 dataset.

Methods: Patients from the French RMD COVID-19 dataset were included in the analysis. COVID-19 diagnosis was established by a positive SARS-CoV-2 PCR test and/or typical symptoms or chest CT scans pattern during the period of the pandemic. Baseline phenotypic characteristics of the RMD including pre-existing ILD prior to the SARS-CoV-2 infection were collected. COVID-19 evolution was characterized as benign (ambulatory care), moderate (hospitalization outside intensive care unit [ICU]) and severe (hospitalization in ICU). Association between the ILD status and the severity and mortality rate of COVID-19 was assessed using multivariable logistic regression adjusted on sex, age, body mass index and diabetes.

Results: By June 26, 2020, 897 patients were included. Pre-existing ILD was reported in 27 patients (3\%): 11 patients with systemic sclerosis, 8 with rheumatoid arthritis, 2 with auto-immune myositis, 2 with mixed connective tissue disease and 4 with other RMD. Among these 27 patients (11 male, mean age $63.1 \pm 16.4 \mathrm{y} / \mathrm{o}, 56.5 \%$ having a usual interstitial pneumonia HRCT pattern), 22 had severe infection. Death related to COVID-19, was observed in 58 patients with RMD without ILD (7.1\%) and in 10 patients with RMD-ILD (37\%). Having a preexisting ILD was found to be independently associated with an increased risk of severe COVID-19 (adjusted OR=7.6 [2.9 - 20.2], $\mathrm{P}<0.001$ ) and an increased mortality rate (adjusted $\mathrm{OR}=12.3[3.8-39.2], \mathrm{P}<0.001$ )

Conclusion: In RMD patients with SARS-CoV-2 infection, preexisting ILD was associated with an increased risk to severe COVID-19 and related mortality. Our findings suggest that RMD-ILD patients should be prioritized for COVID-19 vaccination according to the high morbi-mortality rate during SARS-CoV-2 infection. Disclosure of Interests: Pierre-Antoine Juge Consultant of: BMS, Eric Hachulla Consultant of: Actelion, Bayer, GSK and Pfizer, Grant/research support from: Actelion, Bayer, GSK and Pfizer, Christophe Richez: None declared, Elodie Drumez: None declared, Alain Duhamel: None declared, Raphael Borie: None declared, Philippe Dieudé: None declared

DOI: 10.1136/annrheumdis-2021-eular.2934

\section{\begin{tabular}{|l|l} 
POS1228 & THE ROLE OF CHEST CT IN UNDERSTANDING
\end{tabular} INTERSTITIAL LUNG DISEASE (ILD): SYSTEMIC SCLEROSIS (SSC). VERSUS COVID-19}

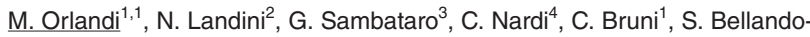
Randone $^{1}$, C. Denton ${ }^{5}$, F. Luppi ${ }^{6}$, B. Ruaro ${ }^{7}$, S. Tomassetti ${ }^{1}$, E. Cavigli ${ }^{8}$, F. Melchiorre ${ }^{9}$, S. Palmucci ${ }^{10}$, S. Guiducci ${ }^{1}$, A. Moggi Pignone ${ }^{1}$, Y. Allanore ${ }^{11}$, A. Bartoloni ${ }^{1}$, M. Confalonieri ${ }^{12}$, G. Cortese ${ }^{13}$, L. Dagna ${ }^{14}$, F. De Cobelli ${ }^{15}$, A. De Paulis ${ }^{16}$, S. Harari ${ }^{17}$, D. Khanna ${ }^{18}$, M. Kuwana ${ }^{19}$, V. Miele ${ }^{8}$, G. Taliani ${ }^{20}$ M. Hughes ${ }^{21}$, C. Vanchieri ${ }^{22}$, S. Colagrande ${ }^{2}$, M. Matucci-Cerinic ${ }^{23} .{ }^{1}$ University of Florence, Department of Experimental and Clinical Medicine, Florence, Italy; ${ }^{2}$ University of Florence, Department of Experimental and Clinical Biomedical Sciences, Radiodiagnostic Unit n. 2, Florence, Italy; ${ }^{3}$ University of Catania, Dept. of Clinical and Experimental Medicine, Catania, Italy; ${ }^{4}$ University of Florence, Department of Experimental and Clinical Biomedical Sciences, Radiodiagnostic Unit n. 2, Florence, Italy; ${ }^{5}$ Centre Head and Professor of Experimental Rheumatology, Experimental Rheumatology, London, United Kingdom; ${ }^{6}$ University of Milano-Bicocca, S. Gerardo Hospital, Respiratory Unit, Monza, Italy; ${ }^{7}$ University Hospital of Cattinara, Department of Pulmonology, Trieste, Italy; ${ }^{8}$ AOUC Careggi, SOD Radiodiagnostica Emergenza-Urgenza, Florence, Italy; ${ }^{9}$ Sant'Andrea Hospital Vercelli, Department of Radiology, Vercelli, Italy; ${ }^{10}$ University of Catania, Department of Medical Surgical Sciences and Advanced Technologies, Catania, Italy; ${ }^{11}$, Descartes University, APHP, Cochin Hospital, Department of Rheumatology A, Paris, Italy; ${ }^{12}$ University Hospital of Cattinara, Department of Pulmonology, Trieste, Italy; ${ }^{13}$ OSPEDALE MARIA VITTORIA e AMEDEO DI SAVOIA, S. C. RADIOLOGIA, Torino, Italy; ${ }^{14}$ IRCCS San Raffaele Scientific Institute, Unit of Immunology, Rheumatology, Allergy and Rare Diseases, Milano, Italy; ${ }^{15}$ RCCS San Raffaele Scientific Institute, Department of Radiology I, Milano, Italy; ${ }^{16}$ University of Naples Federico II, Department of Translational Medical Sciences, Naples, Italy; ${ }^{17}$ Ospedale San Giuseppe MultiMedica, IRCCS, Department of Pulmonology, Milan, Italy; ${ }^{18}$ University of Michigan, Ann Arbor, MI, Department of Medicine, Michigan, United States of America; ${ }^{19}$ Nippon Medical School Graduate School of Medicine, Department of Allergy and Rheumatology, Tokyo, Japan; ${ }^{20}$ Sapienza University, Rome, Department of Translational and Precision Medicine, Rome, Italy; ${ }^{21}$ Sheffield Teaching Hospitals NHS Foundation Trust, Sheffield Teaching Hospitals NHS Foundation Trust, Sheffield, UK, United Kingdom; ${ }^{22}$ University of Catania, Dept. Of Clinical and Experimental Medicine, Catania, Italy; ${ }^{23}$ University of Florence, Dept. Of Clinical and Experimental Medicine, Florence, Italy

Background: COVID-19 pandemic is a global emergency which may overlap on the clinical and radiological scenario of ILD in SSc. In clinical practice, the striking similarities observed at computed tomography (CT) between the diseases make it difficult to distinguish a COVID-19 superinfection from a progression of SSc-ILD.

Objectives: The aim of our study was to identify the main CT features that may help distinguishing SSc-ILD from COVID-19 pneumonia.

Methods: 22 international readers were included and divided in the radiologist group (RAD) and non-radiologist group (nRAD). The RAD group included nonchest RAD and chest-RAD. A total of 99 patients, 52 with COVID-19 and 47 with SSc-ILD, were included in the study.

Results: Fibrosis inside focal ground glass opacities (GGO) in the upper lobes; fibrosis in the lower lobe GGO; reticulations in lower lobes (especially if bilateral and symmetrical or associated with signs of fibrosis) were the CT parameters most frequently associated with SSc-ILD. The CT parameters most frequently associated with COVID- 19 pneumonia were: consolidation (CONS) in the lower lobes, CONS with peripheral (both central/peripheral or patchy distributions) anterior and posterior CONS and rounded-shaped GGOs in the lower lobes. After multivariate analysis, the presence of CONS in the lower lobes $(p<0.0001)$ and signs of fibrosis in GGO in the lower lobes ( $p<0.0001)$ remained independently associated with COVID-19 pneumonia or SSc-ILD, respectively. These two variables were combined in a predictive score which resulted positively associated with the COVID-19 diagnosis, with $96.1 \%$ sensitivity and $83.3 \%$ specificity: 3 different risk class for COVID-19 pneumonia may be identified: high risk for COVID-19 pneumonia (5-9 points); probable overlap COVID-19 pneumonia in SSc-ILD (4 points); low risk for COVID-19 pneumonia (0-3 points)

Conclusion: The CT differential diagnosis between COVID-19 Pneumonia and SSC-ILD is possible and may be fostered in practice by the use of 
radiological score. In the case where an overlap of both diseases is suspected, the presence of consolidation in the lower lobes may suggest a COVID-19 pneumonia while the presence of fibrosis inside GGO may indicate a SSc-ILD.

REFERENCES:

[1] Orlandi M, Landini N, Bruni C, et al. Infection or autoimmunity? The clinical challenge of interstitial lung disease in systemic sclerosis during COVID 19 pandemic. J Rheumatol. 2020 Dec 1: jrheum.200832

[2] Simpson S, Kay FU, Abbara S, et al. Radiological Society of North America Expert Consensus Statement on Reporting Chest CT Findings Related to COVID-19. Endorsed by the Society of Thoracic Radiology, the American College of Radiology, and RSNA [published online ahead of print, $2020 \mathrm{Apr}$ 28]. J Thorac Imaging. 2020;10.1097/RTI.0000000000000524.

[3] Cheng C, Li C, Zhao T, et al. COVID-19 with rheumatic diseases: a report of 5 cases. Clin Rheumatol. 2020;39(7):2025-2029.

[4] Mariano RZ, Rio APTD, Reis F. Covid-19 overlapping with systemic sclerosis. Rev Soc Bras Med Trop. 2020 Sep 21;53:e20200450.

Disclosure of Interests: None declared

DOI: 10.1136/annrheumdis-2021-eular.2967

\section{POS1229 ANTI-MDA5 AND ANTISYNTHETASE ANTIBODIES SCREENING IN SEVERE SARS-COV-2 PNEUMONIA. BE AWARE OF FALSE POSITIVE RESULTS}

A. Gil-Vila ${ }^{1}$, J. Perurena-Prieto ${ }^{2}$, C. Nolla-Fontana ${ }^{1}$, O. Orozco-Galvez ${ }^{1}$, M. Miarons-Font ${ }^{3}$, A. Guillén del Castillo ${ }^{1}$, A. Pacheco-Reyes ${ }^{4}$, E. TralleroAraguás ${ }^{5}$, M. Hernandez-Gonzalez ${ }^{2}, A$. Selva-O'callaghan ${ }^{1}$. 'Vall d'Hebron University Hospital, Internal Medicine, Barcelona, Spain; ${ }^{2}$ Vall d'Hebron University Hospital, Immunology, Barcelona, Spain; ${ }^{3}$ Vall d'Hebron University Hospital, Pharmacology, Barcelona, Spain; ${ }^{4}$ Vall d'Hebron University Hospital, Intensive Care Medicine, Barcelona, Spain; ${ }^{5}$ Vall d'Hebron University Hospital, Rheumatology, Barcelona, Spain

Background: Several reports have shown that severe acute respiratory syndrome coronavirus 2 (SARS-CoV-2) infection may trigger a vigorous immune response that could lead to the appearance of various autoantibodies such as antinuclear antibodies, antiphospholipid antibodies or anti-neutrophil cytoplasmic antibodies, among others. Moreover, the pulmonary involvement in SARSCoV-2 may resemble that of patients with anti-MDA5 positive syndrome or acute form of antisynthetase syndrome.

Objectives: Our aim was to analyse the presence of anti-MDA5 and other myositis-specific autoantibodies such as the antisynthetase antibodies in patients diagnosed with severe acute respiratory syndrome caused by SARS-CoV-2.

Methods: Retrospective observational study performed in a tertiary care center. We included 28 patients admitted to the intensive care unit with severe acute respiratory syndrome, 14 at the onset of the disease (group A) and 14 after 30 days of being treated in an intensive care unit (group B). Chest CT was performed at the admission. We analyzed the presence of anti-MDA5 and antisynthetase antibodies by immunoblot (Euroimmune ${ }^{\circledR}$ ) and in those who were positive we performed a confirmatory test by immunoprecipitation.

Results: All chest CT showed bilateral ground glass pattern. Three out of 14 patients of group A (12 males, $86 \%$, mean \pm SD age $67.1 \pm 12.2$ ) were positive for antisynthetase antibodies ( 2 anti-PL7, 1 anti-Jo1), and 6 out of 14 patients of the group $B(6$ males, $48 \%$, mean \pm SD age $68.7 \pm 8.1)$ were positive to antisynthetase antibodies (2 anti-PL7, 2 anti-PL-12, 1 anti-EJ, 1 anti-OJ+PL7). Immunoblots also show positivity for other myositis-specific or associated antibodies, such as anti-TIF1g, anti-PM75, anti-SAE and anti-SRP. All of these results found by immunoblotting were negative by immunoprecipitation. None of the 28 patients were positive for anti-MDA5 antibodies.

Conclusion: Severe SARS-CoV-2 pneumonia is characterized by ground glass pattern in chest CT, as it is found in anti-MDA5 or antisynthetase syndrome. The positivity of several myositis related autoantibodies showed in immunoblot appears to be more related to the vigorous immune response producing polyclonal immunoglobulins than triggering a real myositis-associated interstitial lung disease. Clinicians must be aware about these false positive results in patients with severe COVID-19 acute respiratory syndrome.

REFERENCES:

[1] Xu Q. MDA5 should be detected in severe COVID-19 patients. Med Hypotheses. 2020; 143:109890.

[2] Giannini M, Ohana M, Nespola B, Zanframundo G, Geny B, Meyer A. Similarities between COVID-19 and anti-MDA5 syndrome: what can we learn for better care? Eur Respir J. 2020; 56:2001618.
[3] Vlachoyiannopoulos PG, Magira E, Alexopoulos H, Jahaj E, Theophilopoulou K, Kotanidou A, Tzioufas AG. Autoantibodies related to systemic autoimmune rheumatic diseases in severely ill patients with COVID-19. Ann Rheum Dis. 2020 Dec;79(12):1661-1663

Disclosure of Interests: None declared

DOI: 10.1136/annrheumdis-2021-eular.3019

\section{POS1230 \\ OUTCOME FOLLOWING COVID-19 INFECTION IN ANTI- NEUTROPHIL CYTOPLASMIC ANTIBODY (ANCA)- ASSOCIATED VASCULITIS}

A. Antovic ${ }^{1}$, B. Lövström ${ }^{2}$, A. Hugelius ${ }^{3}$, O. Borjesson ${ }^{3}$, A. Bruchfeld ${ }^{4}$,

I. Gunnarsson ${ }^{1} .{ }^{1}$ Karolinska Institutet, Department of Medicine, Division of Rheumatology, Unit of Rheumatology, Karolinska University Hospital, Stockholm Sweden; ${ }^{2}$ Karolinska University Hospital, Unit of Rheumatology, Stockhom, Sweden; ${ }^{3}$ Karolinska University Hospital, Unit of Rheumatology, Stockholm, Sweden; ${ }^{4}$ Linköping University, Department of Health, Medicine and Caring Sciences, Linköping, Sweden

Background: Patients with anti-neutrophil cytoplasmic antibody (ANCA)-associated vasculitis (AAV) require immunosuppressive therapy for disease control and reduction of disease relapse and may be at risk for complications during Sars-CoV-2 (COVID-19) infection.

Objectives: To analyze the consequences of COVID-19 in a large cohort of AAV patients regarding occurrence, need of hospitalization, treatment at the intensive care units (ICU), or death.

Methods: Data were retrieved from March 2020 to mid-January 2021 from medical records from the AAV cohort $(n=233)$. Patients diagnosed with granulomatosis with polyangiitis (GPA), microscopic polyangiitis (MPA) or eosinophilic granulomatosis with polyangiitis (EGPA) were included. Data included age, gender, diagnosis, ongoing immunosuppressive medication at onset of COVID-19 or at last follow-up in non-COVID individuals. Renal involvement (ever) and estimated glomerular filtration rate (eGFR) were included. COVID19 was confirmed either by a positive PCR test in the upper airways or by serology. Severe COVID-19 was defined as need of non-invasive ventilation, ICU care, and/or death

Results: The cohort comprised of 172 patients with GPA, 50 with MPA and 11 with EGPA. There were 121 females (52\%). During the study period, 20 patients $(8.6 \%)$ were diagnosed with COVID-19. The median age at data retrieval in all patients was 68 years (21-93), in the COVID-19 group 63 (29-93) and 68.5 (21 90) years in the non-COVID patients.

Fourty-three patients in all (18\%) were hospitalized during the study period of which $11(4.7 \%)$ due to COVID-19 infection. In all, 8 deaths occurred of which 3 were related to COVID-19.

At data retrieval, 110 (47\%) patients were on prednisolone treatment, 10/20 (50\%) in the COVID-19 group and $100(47 \%)$ in the non-COVID-19 group $(p=0.5)$, with significantly higher doses in COVID-19 patients $(p<0.001)$. In patients hospitalized with COVID-19, 6/11 (54.5\%) were on prednisolone, median dose $5 \mathrm{mg} / \mathrm{day}$ (0-50). In the total group $112(48 \%)$ were on disease modifying anti-rheumatic drugs (DMARD) and $64(27.5 \%)$ on rituximab as maintenance therapy. Eight patients were on induction treatment with either cyclophosphamide or rituximab. Of the 20 COVID-19 cases, 8 had severe COVID-19. Of these, 2 were inactive without immunosuppressive treatment, 4 had stable disease with prednisolone (5-7.5 mg/day) in combination with DMARDs, and 2 were active treated with high dose prednisolone (25-50 mg/day) in combination with cyclophosphamide and rituximab $(n=1)$ or rituximab $(n=1)$.

A higher proportion of patients had active AAV $(p=0.03)$ in the severe COVID-19 then in the non-COVID group (10/213 patients)

In the group with the severe COVID-19, 1/8 (12\%) patient had rituximab as maintenance therapy, compared to $61 / 213(28.6 \%)$ in the group of non-COVID-19 patients $(p=0.5)$.

Renal involvement (ever) was present in 144 patients (62\%), in 6 patients $(30 \%)$ with COVID- 19, from which $5(62 \%)$ were in the group of severe COVID-19 patients. Median eGFR did not differ between severe COVID-19 and remaining patients with renal involvement independently of COVID-19 infection.

Conclusion: We found a high rate of severe COVID-19 infection in our cohort of AAV patients which indicates risk for serious complications, especially in patients with active disease and intense immunosuppressive therapy. Maintenance therapy with rituximab did not seem to increase the risk for severe COVID-19. The findings stress the need for continued shielding and early vaccination in AAV patients.

Disclosure of Interests: None declared

DOI: 10.1136/annrheumdis-2021-eular.3020 